\title{
La re-creación de un espacio sin lugar
}

\author{
Mariana Alvarado (1)
}

Resumen: El presente escrito tiene la intención de explicitar un pensamiento latente que devenido en eco advierte su origen en diversas partes y vuelto palabra atraviesa muchos lados para situarse en un amasijo de proyectos que confluyen en la idea intercesora entre dos personas que pretenden hacer de la filosofía una práctica del encuentro dialógico.

\section{LA IDEA INTERCESORA}

El Oráculo surge como idea, años más tarde de aquel encuentro en el que, sobre el programa de Filosofía para niños de Matthew Lipman, tomé contacto con la insistencia de Walter Kohan sobre las preposiciones. La problematización respecto de los límites, alcances y consecuencias de la enseñanza de la filosofía me condujo a sospechar de aquel para y de sus vínculos con los ideales de libertad e igualdad "predominantes" en las sociedades democráticas. Afirmar el con de Filosofía con niños y jóvenes fue el comienzo de una alternativa: la implementación de una Comunidad de Cuestionamiento e Indagación Filosófica fuera del programa lipmaniano e inserta en lo que David Kennedy desarrolla en su "Las cinco comunidades" ( David Kennedy, 1994, p 3-16). Lo que más tarde se haría llamar Pensándonos en el mundo, prometía un espacio circular, susceptible de ocupar diversos lugares, en el que oír las voces de 1@s chic@s del Tercer Ciclo de la Educación General Básica (lo que en el sistema previo a la reforma educativa de 1994 , era el $7^{\circ}$ grado de la escuela primaria y el $1^{\circ}$ y $2^{\circ}$ año de la escuela secundaria o educación media) y de la Educación Polimodal (lo que en el sistema previo a la reforma era $3^{\circ}, 4^{\circ}$ y $5^{\circ}$ de la educación media) daba lugar a identificar en textos filosóficos y no filosóficos las temáticas que una vez en diálogo se problematizarían. El texto - seleccionado por la moderadora - era la excusa para pensar sobre su contenido o sobre lo dicho a partir de él, para que una vez hecha la experiencia pudiéramos pensarnos a nosotros/as en ella. Algo similar era lo que intentamos implementar con Rosa Licata cuando en la tarea teórico-práctica de formar formadores, en la Facultad de Educación Especial y Elemental de la UNCuyo, entre 2002 y 2004, nos abocábamos a experimentar las prácticas que recetaban ciertos textos, transformando las clases magistrales en formas más humanas de diálogo.

Los que integrábamos el grupo que convocaba Adriana Arpini, entre los que recuerdo a Natalia Fischetti, Fabiana Olarieta, María José Guzmán, Carina Quintero, Alejandra Gabriele, Maximiliano López, desde la cátedra de Antropología Filosófica, en la Facultad de Filosofía y Letras de la Universidad Nacional de Cuyo, habíamos hecho la experiencia de pensar con el otro La filosofía en la escuela a partir de dos cuestiones. Por un lado, la necesidad y justificación de la enseñanza de la filosofía en contextos de complejidad creciente, 
el eje que articuló el seminario taller hacia 1999; por otro, valores y transversalidad en la institución educativa, nos mantuvo abocados hacia el 2000.

El contacto con la tradición pragmatista - Charles S. Peirce y John Dewey producto de la lectura de la tesis de licenciatura, El pragmatiso en el proyecto pedagógico de Matthew Lipman: 'Filosofía para niños' Posibilidades y límites del lenguaje de la infancia en la filosofía, elaborada por María del Socorro Jiménez nos dio el punta pié inicial para comenzar a proyectar lo que aun era una idea, pero que ahora se presentaba como intercesora (Cf. Deleuze, 1996, p. 1-5 ) entre Silvana Vignale y yo: hacer de la filosofía una experiencia compartida, de la que nadie es propietario, pero en la que todos somos parte.

\section{LA METÁFORA DEL ECO}

Si no fuera por el metrónomo, el amasijo de beiges, marrones y grises desparramados en "El espejo", de Georges Braque, podría proponerse como la producción pictórica del eco de muchas voces que en el tejido de las palabras se articulan en el diálogo. Cada palabra, una línea; su contenido, un color; cada sonido, una mancha... la presencia del otro, la interferencia entre las rectas; el reconocimiento de la alteridad, la yuxtaposición de tonos; como si derivaran de la interacción que no encima ni enmudece lo diverso, se articulan en el mosaico de inflexiones y regularidades visuales. Formas de reparar en lo que no puede ser visto sino como plural. Aquel aparato, útil para medir los grados de velocidad musical, puede bien ser el símbolo de la intención de medir los tonos del eco que habla su propia voz y los ritmos de lo dicho por el eco del otro; la frecuencia con la que el otro toma su lugar y eleva su voz con la guitarra que se desfigura en el plano, con las palabras que dicen del instrumento musical y de su diseminación. Tiene forma de prisma tal como el cristal que media la diversidad entre los mundos de la vida. El óleo adquiere, en la tela, el perfil de la línea fragmentada por planos. Como si los discursos se enfrentaran en la diferencia de los que oyen y de los que quieren escuchar. Las siluetas que terminan en la homogeneidad de un discurso totalizador quedan reducidas a la mínima expresión del color. Braque permanecerá en la abstracción del cubismo, durante el siglo XX, como la posibilidad de un nuevo lenguaje formal que obliga al espectador a una lectura completamente nueva de la realidad. Un lenguaje que dice de otro modo lo que son las cosas, pero que es la posibilidad de volverse eco y transformarse en otras voces; de leer a través de él nuevas alternativas que, traducidas, posibiliten que las cosas sean y que las palabras digan.

\section{Georges Braque. El espejo}




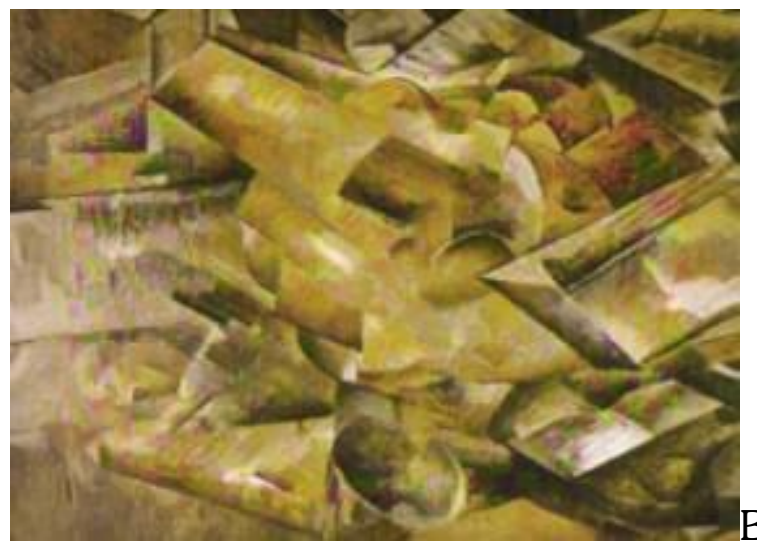

Braque, por lo que en él hay de

figurativo, sin que por ello elimine en su totalidad la presencia objetual. El holandés Piet Mondrian por lo que en él hay de esa ausencia que, como el azar de la memoria, el olvido, trasluce en redes irregulares de colores y líneas sobre la superficie de lo conocido y de lo aún por pensar la imagen reflejada de la posibilidad. El canal en el que predominan las líneas rectas que permiten atender a las curvas provocadas por la tensión del choque de palabras, al igual que su "Composición VII", carecen de otra mediación que las palabras mismas, retículas por las que provocamos una construcción particular en el encuentro con el otro.

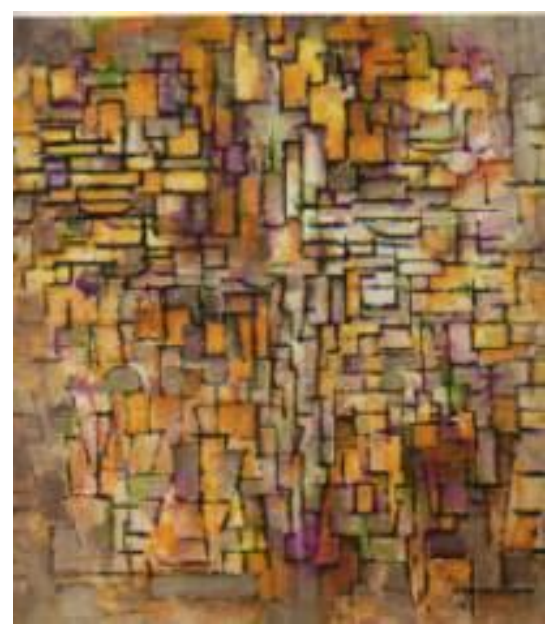

Las telas muestra lo que el ritmo de las palabras articulan: el acontecimiento del diálogo, pero determinan su sentido en la combinación de colores cuando crean las interferencias entre las líneas que lo fijan. El lienzo es apenas la muestra de un instante en el ensayo de voces que se encuentran al pretender escapar del eco del discurso pre - establecido y se sorprenden al dirigirse, por el movimiento de los conceptos, hacia la construcción entre dos del discurso dicho con. Piet Mondrian. Composición VII

\section{LA MIXTURA}

Hace dos años inauguramos en Mendoza, Argentina, El Oráculo. La idea dejó de ser eco para volverse proyecto. En el 2003 lo implementamos en el Teatro Bar los Angelitos. En el 2004 en el Centro Cultural Tajamar. 
La propuesta que intenta ser la alternativa a las certezas, la apertura a la incertidumbre constituye un espacio, sin lugar, con frecuencia semanal, en el que, durante dos horas, se prioriza la indagación, el cuestionamiento y la problematización más que la dogmatización, el afán de dar respuesta y la tematización. Las moderadoras, no alentamos esta prioridad en la soledad de un pensar que monologa. Insistimos en el encuentro con el otro desde la disposición al diálogo.

En un primer momento seleccionamos textos cuyos autores son reconocidos, en el ámbito universitario, como filósofos clásicos. Si proponíamos un espacio para pensar desde la pregunta en el diálogo con el otro, al presentar el texto, surgiría el cuestionamiento. Suponíamos que el texto - cualquiera sea - tenía la cualidad de ser interrogado. Nos equivocamos. El texto no tiene más que lo que ha dejado escrito quien tenía algo que decir. La posibilidad de preguntar supone la disposición de querer hacerlo; no sólo de disputar respecto de respuestas dadas sino también de cuestionar las preguntas que preguntan por aquellas. En cualquier otro caso se trata de repetición filosófica o de posturas a favor o en contra del argumento que se leía. El texto se nos presentaba como el punto de contacto entre los problemas de los filósofos clásicos y el tratamiento no filosófico promovido por no filósofos. Nuestro segundo supuesto era aquel que nos movía a pensar que del texto se pasaría a la experiencia personal. Esto es, si el texto promovía la pregunta poco faltaba para aplicar esa pregunta a los supuestos desde los que nos paramos para ser, escuchar, hacer, sentir, decir, etc. Dejar fuera aquel punto de contacto con los clásicos, los modernos, los contemporáneos, y hacer de la filosofía el dispositivo por el cual trataríamos nuestros problemas y no los de aquellos. Dejar de ser no filósofo para abrir un nuevo comienzo signado por el pensar filosóficamente lo no filosófico.

Si hablamos de una disposición, ¿cómo promoverla? Pensamos en catalogar los textos que poníamos a prueba. Diferenciamos entre textos problemáticos y textos temáticos. Esto es, por un lado, aquellos que presentan un problema, dilema, encrucijada, acertijo, etc. susceptible de a resolver y por otro aquellos que presentan argumentos a favor o en contra de una postura. Suponíamos entonces que el planteo del problema permitiría pensar alternativas diversas. Nuestra búsqueda se orientaba, por tanto, a textos problemáticos. Esto ya implicaba, a nuestro modo de ver, un pensar propio en tanto debían articularse diversas respuestas. Consideramos radical la concientización de la posibilidad de articular alternativas múltiples ya que esto no sólo permitiría valorar diversas perspectivas independientemente del acuerdo que se tenga con ellas sino además presentar la propia como una posible entre muchas. Tener algo que decir y estar dispuesto a que se haga público ya era mucho para los tantos que escuchaban y los pocos que siempre hablaban. El texto no dejaba de ser el medio por el que se presentaba un problema ajeno.

Nos gustaba jugar con la idea de que el procedimiento sería puesto en cuestión. Reservábamos los últimos minutos del café para llevar a cabo una auto y/o metacognición Esto es, pensar lo que hicimos juntos y lo que cada uno hizo por separado. Autoevaluarse, auto-corregirse, auto-gobernarse ¿auto-resignarse? Las 
miradas se dirigían a la propuesta y se hablaba de nuestro hacer como si no fuera nuestro... no existía sentido perteneciera. Varios encuentros transitamos para que alguien preguntara - "¿por qué utilizamos textos de filósofos?" - y para que alguien propusiera - "¿podemos escribir nuestros propios textos?" -. Fue entonces cuando de la mirada racional hacia El Filósofo nos situamos en la mirada viseral de los filósofos, cuando de la lectura de la Filosofía pasamos a su práctica. Cada uno tenía entonces, la posibilidad reflexionar durante una semana entorno a un tema y poner en un papel, si quería o no, lo que diría al respecto. Pocos fueron los que escribieron preguntas. La mayoría presentó una tesis con pocos argumentos para defenderla. Entonces los encuentros se redujeron a la presentación de tesis que enfrentadas, lejos estaban de ponerse en cuestión una a la otra o de dar lugar para falsarse a sí misma.

Extraño a la microeconomía de los tiempos, las tareas, las acciones, las palabras, los comportamientos, propia de instituciones en las que se determina lo que se espera de él/ella - los enfermos, los trabajadores, los hijos, los empleados, los anormales, los estudiantes, los soldados, los civiles - y en las que normatizar y sancionar cuando los resultados no son los previstos se vuelven la estrategia para economizar la distribución de los premios y los castigos, concebimos El Oráculo como un espacio abierto al encuentro en el que se construye comunitariamente la ciudadanía. Alejado de instituciones destinadas a adiestrar, instruir, enderezar, encaminar, corregir, formar... educar...se presenta como una alternativa a ellas e incluso como la resistencia desde la que es posible reconstruir otras relaciones con tales instituciones, con las personas que las dirigen, con las prácticas que implementan, con las estrategias que someten, con los sujetos que las padecen.

Concebimos la filosofía como una práctica que no podría existir si no existiéramos, que en cuanto ejercicio colectivo del pensamiento precisa de un querer, es decir de "un tener" la intención de practicarla, de "un cómo" hacerlo, en diálogo con el otro y, de "una atribución" de sentido a lo que se hace en ese hacer y a lo que se hará a partir de él. La filosofía es inútil en cualquier otro caso porque no tiene lugar para ser lo contrario.

Aunque la pregunta con la explicitación de los signos de interrogación parezca no tener terreno teórico donde gestarse porque a primera vista parezca que nadie ha querido dárselo; las zonas del perspectivismo posibilitaron las condiciones para hablar otras lenguas en las que se resuelven las tensiones entre pertenencia y participación a partir de la afirmación del otro como diverso, de la diversidad como lo que nos hace alteridad, de la universalidad de los derechos de todos, de la diferencia de los derechos de grupo, de las condiciones mínimas de igualdad de grupo en diálogo. La diversidad ha tenido su lugar en el encuentro entre extranjeros como la condición que permite la conciliación entre re-creación, cultura y turismo. Allí se hizo presente la pregunta en cuanto apertura al otro. Se escuchaba ¿cómo es posible que piense así? Se decía _Es que es chileno. Se replicaba _ pero ¿cómo? ¿el origen étnico justifica la posibilidad de escucha de lo distinto? Se continuaba _ ¿él puede decir lo que quiere porque no es argentino? y... los argentinos no siempre dicen lo que quieren y si lo dicen... -Se abría hacia otros esferas _ ¿cuáles son los riesgos de insistir en las diferencias culturales? - Una 
comunidad que tiene la intención de encontrarse y explorar los riesgos de relacionarse con los otros ya no para la reproducción de lo conocido sino más bien para la creación de la posibilidad puede, entre otras alternativas, someterse al encantamiento. El extranjero se hizo presente como conquistador. Pero su hechizo conquistador aparece allí donde se ha permitido la complicidad de las relaciones instrumentales. La apertura da lugar, sin restricción, a lo que emerja... ¿cómo prevenir posturas autoritarias, devastadoras, absolutistas, totalitarias, elitistas, mutiladoras, tiránicas, dictatoriales, devastadoras, etc sin caer en autoritarismos, totalitarismos, elitismos y todos los ismos que puedan aventurarse?

Madrileños, peruanos, yanquis... llegaban al café llamados por la recreación del "atractivo turístico" que permitía "cultivarse". Tal fue el caso de José, quien se presentó al finalizar uno de los últimos encuentros del ciclo 2004. En el momento en el que nos disponíamos a pensar la práctica del día y nuestra puesta en ejercicio en ella, José nos cuenta que viene de Estados Unidos, que es filósofo, que está asqueado de la política yanqui y que vive en Mendoza desde hace tres años. Apenas acusa recibo de que es difícil entenderle. Habla español, escucha algo y atiende poco. Lo acompaña su traductora personal, quien parecía estar allí por el lazo afectivo más que por una cuestión laboral. Continúa _... aquí no se piensa muy distinto de como se lo hace en los bares de donde vengo... El hecho es que Juan se presenta como Filósofo - con título y todo - y cuenta el tema de su tesis de doctorado "física cuántica" a lo que le siguió un prolongado - ioohh!!!! Proveniente de quienes dejaron de ser filósofos parlantes para formar parte del auditorio. Juan propone un tema. Lo escribe en el temario y todos votan su propuesta. Sí, todos. ¿Cuál fue el tema? "La física cuántica y Jesús" i¿?!

Cuando pensábamos que los filósofos hacíamos filosofía sobre lo no filosófico aparece La Filosofía para distraernos de la filosofía. La Filosofía entonces nos distrae de lo cotidiano y nos vuelve no filósofos ( Ceppas, 2005, pp 50-54). Pensar lo acontecido requirió, de parte de las moderadoras, no sólo la puesta en juego del rol que hasta el momento consideraban alternar con los dialogantes sino además la necesidad de pensar los límites, condiciones y posibilidades de los canales de circulación del discurso, de los lugares que ocupan los sujetos por la emisión de las realidades mentadas en los conceptos y de las actitudes que contribuyen o no a la des-integración y al no-reconocimiento del alteridad en su diversidad.

Desde entonces, enfocamos nuestro análisis en dos instancias. Por un lado, El Oráculo que en cuanto Café Filosófico fue adoptado como fenómeno turístico independiente de la comercialización del té, las masitas, la Andes (cerveza propiamente mendocina) bien helada o el maní - evidencia una práctica susceptible de propiciar el encuentro, el reconocimiento, el diálogo, el intercambio, la concertación entre sujetos que se afirman en la diversidad. Dar lugar a la reflexión acerca de lo que nos preocupa trae consigo la necesidad de atender a las interpretaciones acerca de los valores, normas y fines que están a la base de lo que se considera prioridad a ser pensada. Disponerse a pensar con el otro propicia la interacción cultural y, en tal sentido las prácticas que promueven la interculturalización y/o transculturalización de la diversidad habilitan para vivir entre la alteridad y sin renunciar a su identidad. Por otro lado, la conquista. En esa 
instancia no podemos dejar la historia de los pueblos latinoamericanos de lado - el viejo debate civilización o barbarie - para conocerla, reconocerla, recordarla y recrearla o traducirla o para olvidarla pero siempre para pensarla. Un proceso en el que el entrecruzamiento de las interpretaciones propiciaría la experiencia de dessujetarse, re-sujetarse y re-ubicarse contextualmente; atendería a la posibilidad de no eliminar la diversidad, de mantener intactas las identidades y su autonomía, de pensar el conflicto entre valores éticos universales y bienes culturales; proveería los medios inteligibles para identificar los escenarios que unen a los sujetos autoimplicados en la construcción de una ciudadanía que precisa de un estado multiétnico, plurinacional, diferenciador e igualitario.

Nos proyectamos hacia el 2005 con la idea de salir al encuentro de un nuevo lugar para El Oráculo. El espacio en el que se produce, reproduce, recrea, traduce, construye, significa, hará explotar la posibilidad de pensar a partir de la tradición latinoamericana en el encuentro dialógico de conjuntos más o menos numerosos de indagadores políglotas, en un tiempo estimado de dos horas, con una intensidad impredecible y con consecuencias poco medibles.

Todo un mosaico de variaciones dispuestas a ser fijadas en la tela que cada uno esté dispuesto a pintar.

\section{BIBLIOGRAFÍA}

Ceppas, Filipe. "Sobre las prácticas filosóficas extra-académicas". En: Novedades Educativas. La enseñanza de la filosofía. Argentina, no 169, Año 17, 2005, pp 50 54.

Deleuze, G. "Los intercesores" En: Conversaciones de Pilles Deleuze. Valencia, Pretextos, 1996. Formato word.

Kennedy, David "The Five Communities", Analytic Teaching, vol 15, n 1, 1994, pp. 3-16.

(1) Es profesora de grado universitario en filosofía, por la Facultad de Filosofía y Letras de la UNCuyo, Mendoza, Argentina. Jefa de Trabajos Prácticos en la Cátedra de Antropología Filosófica de la Carrera de Psicología, Universidad de Congreso. Practica la docencia en el Tercer Ciclo de la Educación General Básica y en la Educación Polimodal. Investigadora junior ad honorem de la Sec yTunc. Creadora del proyecto café filosófico El Oráculo, que actualmente implementa junto a Silvana Vignale en el Museo Municipal de Arte Moderno en la Ciudad de Mendoza, Argentina.

Email: alvaradom@ucongreso.edu.ar 